\title{
Blind Signal Processing for Medical Sensing Systems with Optical-Fiber Signal Transmission
}

\author{
Namyong Kim and Hyung-Gi Byun ${ }^{+}$
}

\begin{abstract}
In many medical image devices, dc noise often prevents normal diagnosis. In wireless capsule endoscopy systems, multipath fading through indoor wireless links induces inter-symbol interference (ISI) and indoor electric devices generate impulsive noise in the received signal. Moreover, dc noise, ISI, and impulsive noise are also found in optical fiber communication that can be used in remote medical diagnosis. In this paper, a blind signal processing method based on the biased probability density functions of constant modulus error that is robust to those problems that can cause error propagation in decision feedback (DF) methods is presented. Based on this property of robustness to error propagation, a DF version of the method is proposed. In the simulation for the impulse response of optical fiber channels having slowly varying dc noise and impulsive noise, the proposed DF method yields a performance enhancement of approximately $10 \mathrm{~dB}$ in mean squared error over its linear counterpart.
\end{abstract}

Keywords: Optical links, Dc noise, Impulsive noise, Biased error, Constant modulus, Decision feedback

\section{INTRODUCTION}

The Gaussian noise model has been widely accepted in most of the studies on the effects of noise on the performance of optimization strategies, but when considering dc offset or biased noise (dc noise), some significant differences arise [1]. In electroencephalogram (EEG) recording systems, high electrode impedances between recording electrodes and living skin tissue can increase the low-frequency noise impacted by electrode impedance. This slowly varying dc noise will cause enormous variance in the measurement of effective refractory period (ERP) if it's not cancelled [2]. In addition, dc noise is also a problem in endoscopy using optical fibers. The amount of light that the CCD sensor of an endoscope receives may vary greatly depending on the position of the distal end of the endoscope. This variation in the amount of light may appear as varying dc noise and affect the displayed image [3].

Division of Electronics, Information \& Communication Engineering, Kangwon National Unversity, Samcheok, Gangwon-Do, 245-711, Republic of Korea ${ }^{+}$Corresponding author: byun@kangwon.ac.kr (Received : Nov. 28, 2013, Revised: Jan. 2, 2014, Accepted: Jan. 7, 2014)

This is an Open Access article distributed under the terms of the Creative Commons Attribution Non-Commercial License(http://creativecommons.org/ licenses/bync/3.0) which permits unrestricted non-commercial use, distribution, and reproduction in any medium, provided the original work is properly cited.
In recent capsule endoscopy systems, an on-line wireless link to the computer is designed for early diagnosis and facilitating patient's movement. However, in the indoor environments, the wireless link is susceptible to noise from other wireless devices and multipath fading that causes inter-symbol interference (ISI) in the received signal. Moreover, in an indoor wireless link, impulsive noise from various indoor electronic devices with electromechanical switches is present [4,5]. The impulsive and slowly varying dc noises can be considered crucial in bio-sensing or medical diagnosis systems, resulting in transmission errors that are unacceptable in communication systems dealing with medical information, these noise must be cancelled [6].

Optical fiber communications are used in medical imaging, remote bio-sensing, plumbing inspections, sewer lines and in Internet signals. In optical communications, high-speed data transfer is often limited by signal distortion, which is mainly caused by broadening of pulses that results $\mathrm{n}$ ISI. To cope with ISI, adaptive algorithms based on the mean squared error (MSE) criterion have been widely chosen [7,8]. Unsupervised equalization algorithms operate without the need for training data for starting or restarting after a communications breakdown and most blind equalization algorithms utilize the nonlinearity of the equalizer output for updating weights. As one of the well-known blind algorithms based on the MSE criterion, the constant modulus algorithm (CMA) minimizes the average power of the constant modulus error (CME) between the instant output power 
and source constant modulus [9]. One of the drawbacks of this algorithm is that its performance is not satisfactory because of the limits of the MSE criterion.

Unlike the MSE criterion that uses squared error, information theoretic learning (ITL) methods are based on a combination of a nonparametric probability density function (PDF) estimator and the concept of information potential (IP) [10]. As one of the ITL methods, the minimum error entropy (MEE) criterion has shown superior performance when compared with the MSE criterion in supervised channel equalization applications [11]. Another ITL criterion, the Euclidian distance (ED) between two PDFs that contains only quadratic terms to utilize the tools of information potential has been successfully applied to biomedical classification problems [12].

All these criteria are in Gaussian noise environments. Recently, to cope with impulsive noise, an autocorrelation function of kernel-transformed random processes called, correntropy has been proposed for correlative signaling systems [13]. However, the correntropy blind algorithm does not yield acceptable performance in independent identically distributed signaling methods that most communication systems employ and in dc bias noise environments.

For optical communication systems contaminated with dc noise, a blind signal processing criterion based on the CME and IP has been introduced in [14]. The criterion utilizes the ED between the biased PDF of CMEs and the Dirac-delta function located at zero on the CME axis.

In this study, we investigate whether the method in [14], which was originally designed for canceling dc noise and ISI, is robust to impulsive noise as well. Because impulsive noise produces bursts of incorrect decisions that can cause significant error propagation in decision feedback equalizer (DFE), DFE structures can be employed for residual ISI cancellation. On the bases of an analysis of the ED between the biased PDF of CMEs and the Dirac-delta function, we propose the DFE version of the method that is not only robust to impulsive and dc noises but also capable of effectively canceling overall ISI from multipath or optical-fiber dispersion.

\section{METHODS}

\subsection{Equalizer cost functions for cancelling noise and ISI}

Generally, ISI exists in all optical communication systems, and equalizers are ideal devices to compensate for ISI from dispersion along with numerous other distortion effects [15]. A general tapped delay-line (TDL) equalizer where tap weights are adjusted by some adaptive algorithms mostly based on the MSE criterion, is commonly used. The adaptive equalizer will then handle time-varying dispersion and other linear slow varying distortion effects. However, blind equalizers that do not require training sequences for calculating output error can utilize the CME instead.

The CME at time $\mathrm{k} e_{C M E, k}$ is defined as the difference between the instant power of output $y_{k}$ and the constant modulus $R_{2}$, i,e, $e_{C M E, k}=\left|y_{k}\right|^{2}-R_{2}$, where $R_{2}=E\left[\left|A_{k}\right|^{4}\right] /\left(E\left[\left|A_{k}\right|^{2}\right]-R_{2}\right)$ is determined on the basis of the transmitted symbol points $A_{k}$ [16]. The MSE criterion for blind algorithms based on the CME is

$$
M S E=E\left[e_{C M E, k}^{2}\right]=E\left[\left(\left|y_{k}\right|^{2}-R_{2}\right)^{2}\right]
$$

With biased dc noise adaptive systems may produce shifted error. In this case, the biased error PDF in (3) can be moved along the axis by controlling the variable $\tau$. In order to move this PDF toward the origin, an optimization criterion has been proposed in [14], in which the ED between the biased PDF of CME $f_{C M E}(e-\tau)$ and the Dirac-delta function $\delta(e)$ located at zero on the CME axis is minimized.

$$
\begin{aligned}
& E D=\int\left[f_{E}(e-\tau)-\delta(e)\right]^{2} d e= \\
& \int f_{C M E}^{2}(e-\tau) d e-2 \int f_{C M E}(e-\tau) \delta(e) d e+\int \delta^{2}(e) d e
\end{aligned}
$$

Using the kernel density estimation method in [17] with a block of CME samples $\left\{e_{C M E, k}, e_{C M E, k-1}, \ldots, e_{C M E, i}, \ldots, e_{C M E, k-N+1}\right\} \mid$ and the zero-mean Gaussian kernel $G_{\sigma}\left(e-e_{C M E, i}\right)=\frac{1}{\sigma \sqrt{2 \pi}} \exp \left[\frac{-\left(e-e_{C M E, i}\right)^{2}}{2 \sigma^{2}}\right]$, the PDF of the CME $f_{C M E}(e)$ can be given by

$$
f_{C M E}(e)=\frac{1}{N} \sum_{i=k-N+1}^{k} G_{\sigma}\left(e-e_{C M E, i}\right)
$$

By employing a bias variable $\tau$, the cost function to be minimized can be given as

$$
\left.\operatorname{Cost}=\frac{1}{N^{2}} \sum_{i=k-N+1 j=k-N+1}^{k} \sum_{\sigma \sqrt{2}}^{k} G_{C M E, i}-e_{C M E, j}\right)-2 \frac{1}{N} \sum_{i=k-N+1}^{k} G_{\sigma}\left(e_{C M E, i}+\tau\right)
$$

For convenience, this cost function (4) as proposed in [14], will be referred to as minimum ED for biased CME (MED-BCME) in this paper.

\subsection{Proposed decision feedback method}

In an environment with strong noise, most blind learning 
methods frequently produce incorrect decisions that can cause error propagation when a decision feedback structure is employed [16]. The DFE comprises a feed-forward filter for the input signal and a feedback filter for decided symbols $\hat{d}_{i}$, and the DFE output is

$$
y_{i}=\mathbf{X}_{i}^{T} \mathbf{W}_{i}^{F}+\hat{\mathbf{D}}_{i-1}^{T} \mathbf{W}_{i}^{B}
$$

The feed-forward filter consists of a feed-forward weight vector $\mathbf{W}_{i}^{F}=\left[w_{i, 0}^{F}, w_{i, 1}^{F}, w_{i, 2}^{F}, \ldots, w_{i, P-1}^{F}\right]^{T}$ and input vector $\mathbf{X}_{i}=$ $\left[x_{i}, x_{i-1}, \ldots, x_{i-P+1}\right]^{T}$. The feedback weight vector is $\mathbf{W}_{i}^{B}=$ $\left[w_{i, 0}^{B}, w_{i, 1}^{B}, w_{i, 2}^{B}, \ldots, w_{i, Q-1}^{B}\right]^{T}$ and the feedback filter receives a sequence of decisions on previously decided symbols and a constant $c$ as $\hat{\mathbf{D}}_{i-1}=\left[d_{i-1}, d_{i-2}, \ldots, d_{i-Q-2}, c\right]^{T}$. This feedback filter section is used to remove residual ISI.

In the cost function (4) composed of two Gaussian kernels, the kernel $G_{\sigma}\left(e_{C M E, i}+\tau\right)$ is an exponential decay function of the distance between two error locations so that the excessively long distance between two error samples, $e_{C M E, i}-e_{C M E, j}$ induced by impulsive noise becomes a negligibly small value. Moreover, the Gaussian kernel $G_{\sigma}\left(e_{C M E, i}+\tau\right)$ plays two roles in the minimization of the cost function. One is that with an appropriately adjusted value of $\tau$, the shifted error samples induced by biased dc noise can be relocated to be centered at zero. The other is that the kernel causes the cost function to be insensitive to large error samples. This immunity to $\mathrm{dc}$ and impulsive noises allows the system to prevent incorrect decisions and error propagation problems when decision feedback structures are employed.

When we define $y_{i}^{R}=\sum_{p=0}^{P-1} w_{i, p}^{F} x_{i-p}+\sum_{q=0}^{Q-1} w_{i, q}^{B} \hat{d}_{i-q-1}$, the output (5) can be rewritten as

$$
y_{i}=y_{i}^{R}+w_{i, Q}^{B} c
$$

Then the CME becomes

$$
e_{C M E, i}=\left|y_{i}^{R \mid}+w_{i, Q}^{B} c\right|^{2}-R_{2}
$$

Substituting (7) into the cost function (4), we have

$$
\begin{aligned}
\operatorname{Cost} & \left.=\left.\frac{1}{N^{2}} \sum_{i=k-N+1 j=k-N+1}^{k} \sum_{\sigma \sqrt{2}}^{k} G_{i}\left|y_{i}^{2}-\right| y_{j}\right|^{2}\right) \\
& -\frac{2}{N} \sum_{i=k-N+1}^{k} G_{\sigma}\left(\left|y_{i}^{R}+w_{i, Q}^{B} c\right|-\left(R_{2}-\tau\right)\right)
\end{aligned}
$$

When the cost function (8) is minimized, the second term

$$
\frac{2}{N} \sum_{i=k-N+1}^{k} G_{\sigma}\left(\left|y_{i}^{R}+w_{i, Q}^{B} c\right|^{2}-\left(R_{2}-\tau\right)\right) \text { is maximized so that }\left|y_{i}^{R}+w_{i, Q}^{B} c\right|^{2}
$$

approaches the constant $R_{2}-\tau$. Namely, the variable $\tau$ can be controlled to cancel the bias of the error PDF with the aid of $w_{i, Q}^{B} c$. Further, large argument values due to impulsive noise are discarded with the exponentially decaying characteristics of Gaussian kernel. This analysis indicates that, in an appropriate filter structure, the cost function (8) has immunity to impulsive and dc noises as well as the ability to cancel ISI.

By minimizing the cost function Cost with respect to the filter weights using the gradient descent method, the forward and backward filter weights can be recursively adjusted. The forward section gradient is evaluated as

$$
\begin{aligned}
& \frac{\partial \operatorname{Cost}}{\partial \mathbf{W}^{F}}=\frac{1}{2 \sigma^{2} N^{2}} \\
& \sum_{i=k-N+1}^{k} \sum_{j=k-N+1}^{k} G_{\sigma \sqrt{2}}\left(\left|y_{i}\right|^{2}-\left|y_{j}\right|^{2}\right)\left(\left|y_{j}\right|^{2}-\left|y_{i}\right|^{2}\right)\left(y_{i} \mathbf{X}_{i}^{*}-y_{j} \mathbf{X}_{j}^{*}\right) \\
& +\frac{1}{\sigma^{2} N} \sum_{i=k-N+1}^{k} G_{\sigma}\left(\left|y_{i}\right|^{2}-\left(R_{2}-\tau\right)\right)\left(\left|y_{i}\right|^{2}-\left(R_{2}-\tau\right)\right) y_{i} \mathbf{X}_{i}^{*}
\end{aligned}
$$

Similarly, the backward section gradient becomes

$$
\begin{aligned}
& \frac{\partial \operatorname{Cos} t}{\partial \mathbf{W}^{B}}=\frac{1}{2 \sigma^{2} N^{2}} \\
& \sum_{i=k-N+1}^{k} \sum_{j=k-N+1}^{k} G_{\sigma}\left(\left|y_{i}\right|^{2}-\left|y_{j}\right|^{2}\right)\left(\left|y_{j}\right|^{2}-\left|y_{i}\right|^{2}\right)\left(y_{i} \hat{\mathbf{D}}_{i-1}^{*}-y_{j} \hat{\mathbf{D}}_{j-1}^{*}\right) \\
& +\frac{1}{\sigma^{2} N} \sum_{i=k-N+1}^{k} G_{\sigma}\left(\left|y_{i}\right|^{2}-\left(R_{2}-\tau\right)\right)\left(\left|y_{i}\right|^{2}-\left(R_{2}-\tau\right)\right) y_{i} \hat{\mathbf{D}}_{i-1}^{*}
\end{aligned}
$$

where $N \geq P$ and $N \geq Q$.

Using the gradients (9) and (10), the weight vectors for the feed-forward and feedback sections are updated with a step size $\mu$ as follows;

$$
\begin{aligned}
& \mathbf{W}_{k+1}^{F}=\mathbf{W}_{k}^{F}-\mu \frac{\partial \operatorname{Cos} t}{\partial \mathbf{W}^{F}} \\
& \mathbf{W}_{k+1}^{B}=\mathbf{W}_{k}^{B}-\mu \frac{\partial \operatorname{Cos} t}{\partial \mathbf{W}^{B}}
\end{aligned}
$$

In this paper, the proposed DF algorithm will be referred $t o$ as decision feedback MED-BCME (DF-MED-BCME).

\section{RESULTS AND DISCUSSION}

For this simulation, an impulse response of the optical fiber channel is chosen from the results in [18] as discussed below.

This channel model includes a transmitter impulse response, wired or wireless channel impulse response, and receiver matched-filter impulse response [19]. 


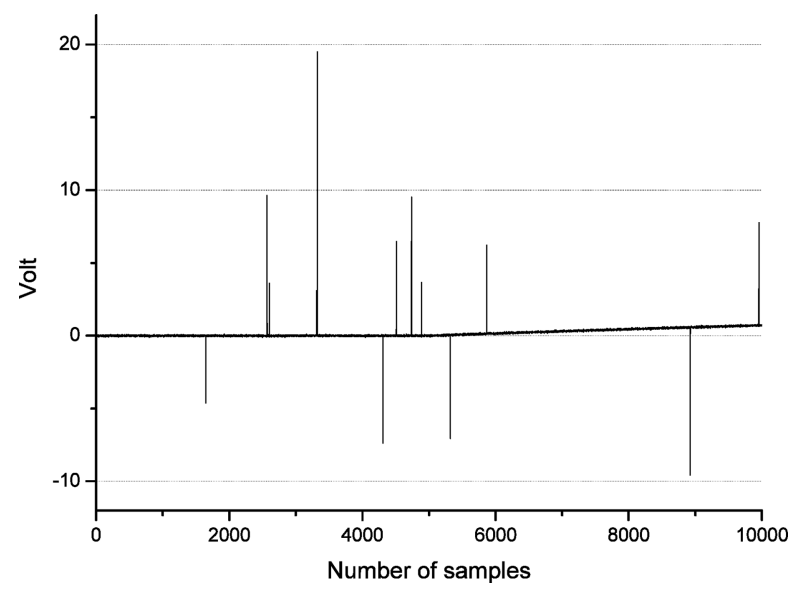

Fig. 1. Example of overall noise with impulsive and slowly varying dc noise.

$$
H(z)=0.2574+0.6905 z^{-1}+0.5478 z^{-2}+0.3440 z^{-3}+0.1962 z^{-4}
$$

A binary symbol $\left(A_{1}=1, A_{2}=-1\right)$ is transmitted through the channel (13), and then impulsive and dc biased noises are added. The zero-mean white impulsive noise to be added to the channel output is generated according to the work [13] with the sum of the variance in the impulse and background noise being 50 and the impulse incident rate 0.002 . The variance in the background white noise is set to be 0.001 . Static dc noise can be removed by simple analog or digital filters, so in this simulation, a dc noise that varies slowly with time is added from sample number 5000 as shown in Fig. 1.

The numbers of feed-forward and feedback filter weights are $P=11$ and $Q=4$, respectively. The linear algorithms have a corresponding number of 15 weights. The constant modulus $R_{2}=1$ because the transmitted symbol is $\left(A_{1}=1, A_{2}=-1\right)$. We test the CMA, correntropy [13], their DF versions (DF-CMA and DF-correntropy), MED-BCME, and DF-MED-BCME algorithms by comparing their MSE convergence performance. The step-size of the CMA is 0.000005 and the data-block size $N$ is 30 . The step size and kernel size of correntropy are 0.002 and 4.2, respectively. The step size, constant $c$, and kernel size of both MED-BCME and DF-MED-BCME are set to be 0.02, 2.0 and 2.0, respectively. These parameters are selected to have the lowest steady-state MSE for each algorithm.

In the case of slowly varying dc noise along with impulsive noise, steady state MSE of MED-BCME and DF- MED-BCME show no sensitivity to the influx of slowly varying dc noise as shown in Fig. 2. The conventional CMA and correntropy converge slowly under non-Gaussian noise and ISI from the fiber

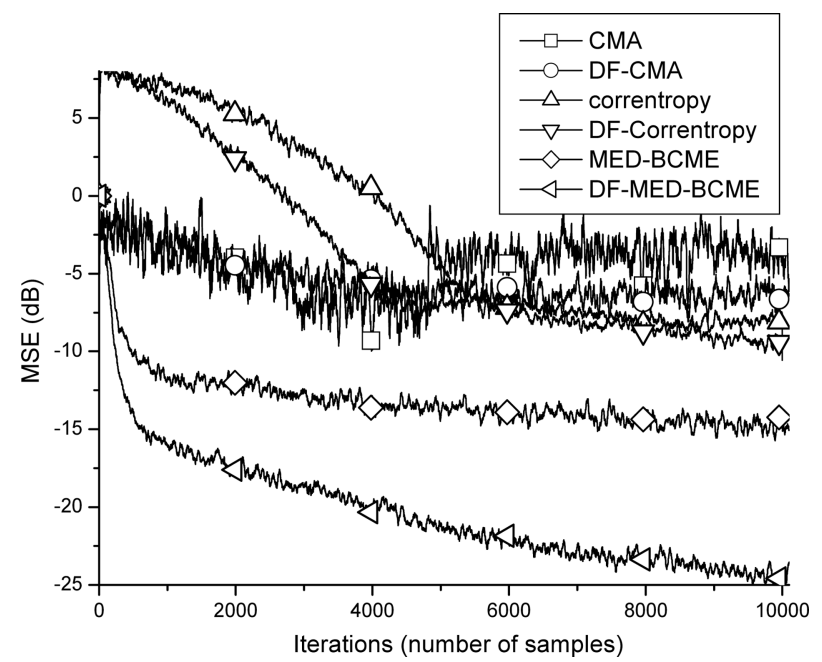

Fig. 2. MSE convergence performance under ISI and non-Gaussian noise. (Note that gradually increasing dc noise is added to the impulsive noise from sample number 5000).

optic systems. From the sample time as the dc noise increases gradually increase, the MSE curves of the CMA and DF-CMA increase and converge at $-2 \mathrm{~dB}$ for the CMA and $-3 \mathrm{~dB}$ for the DF-CMA. Correntropy appears stays at about $-6 \mathrm{~dB}$ for linear and $-8 \mathrm{~dB}$ for DF-correntropy. On the other hand, the MED-BCME type algorithms reach $-14 \mathrm{~dB}$ for the MED-BCME and $-24 \mathrm{~dB}$ for the DF-MED-BCME. This result indicates that the DF version of MED-BCME is significantly effective in cancelling the overall ISI from fiber optic systems owing to the robustness of the MED$\mathrm{BCME}$ to impulsive and dc noises and its error propagation blocking capabilities.

For a rigorous performance evaluation in digital signal transmission, the average symbol error rate (SER) is commonly used. However, as can be seen in Fig. 3 (for an output signal with impulsive noise) and Fig. 4 (for a time region afflicted by impulse noise for a closer inspection) after convergence, symbol errors are mainly caused by the high voltage of impulses, which are generated randomly at an occurrence rate specified in the simulation. Therefore, Fig. 5 shows that after convergence, the output samples of the proposed equalizer have no symbol errors in the time regions having no impulsive noise. The value of $-25 \mathrm{~dB}$ steady state MSE under the given background white-noise variance (0.001) indicates that in the case without impulsive noise the SER can be almost zero as observed in Fig. 5. Moreover, the proposed algorithm is not perturbed by the strong impulses and shows showing correctly recovered estimation immediately after the impulses shown in Fig. 4. In addition, the dc biased noise is completely cancelled by the proposed algorithm in shown as Figs. 4 and 5. 


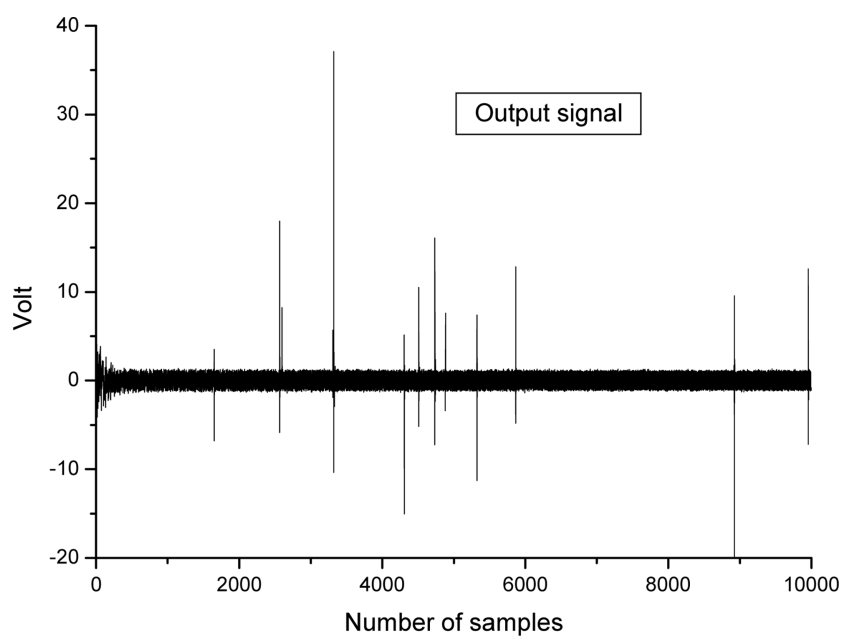

Fig. 3. Output signal of the proposed equalizer under ISI and nonGaussian noise.

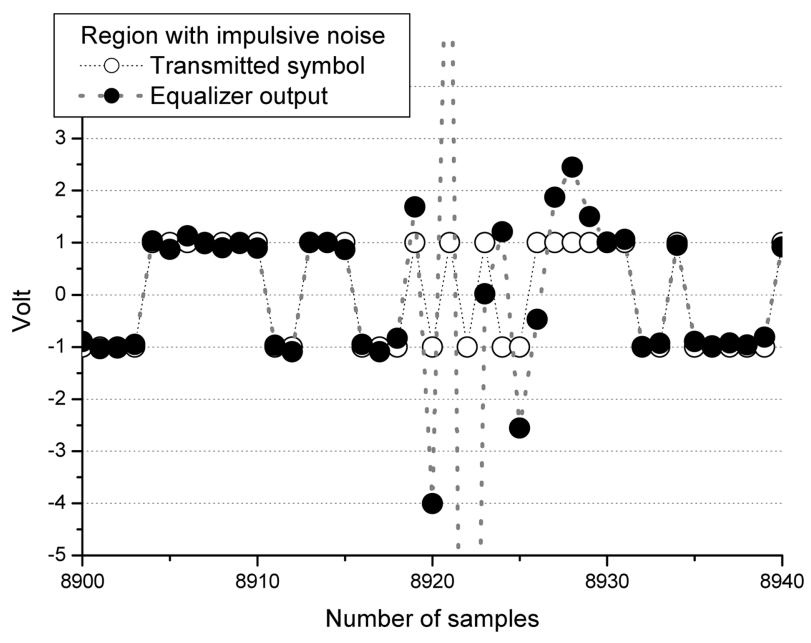

Fig. 4. After-convergence output signal in a region with impulsive and $\mathrm{dc}$ noises.

\section{CONCLUSION}

From EEGs to endoscopes, many medical devices suffer from dc noise that prevents a doctor from making a correct diagnosis. In recent wireless capsule endoscopy systems, multipath fading occurs through various tissues or organs and wireless links in indoor environments. This multipath fading induces ISI in the received signal, and in indoor wireless links, impulsive noise occurs from various indoor electronic devices. These ISI and nonGaussian noise are also found in optical fiber communication systems that can be used in remote medical diagnosis and operations. In this paper, the robustness of the blind equalization method based on the biased PDF of CMEs to impulsive noise to

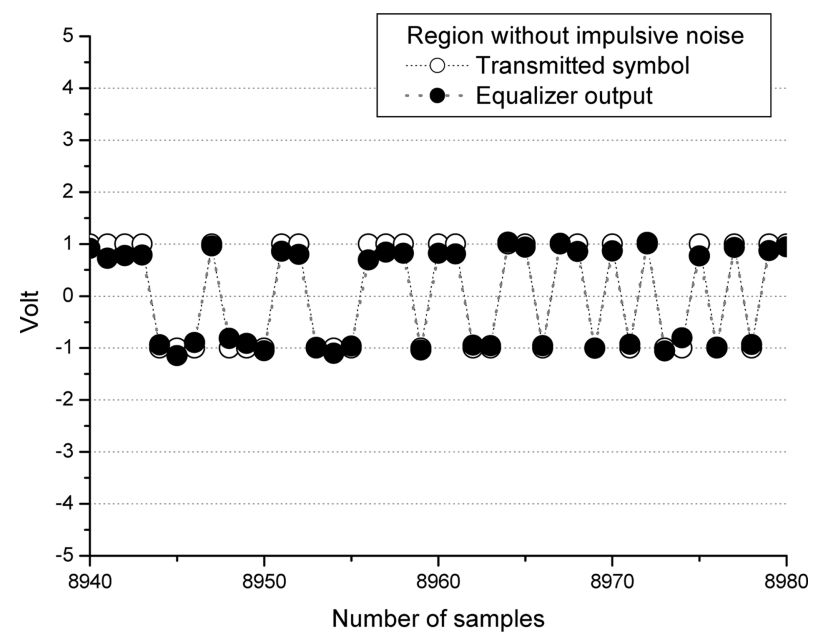

Fig. 5. After-convergence output signal in a region without impulsive noise.

effectively cope with ISI under dc and impulsive noise is investigated. Based on the analysis, a DF version of the method is proposed because the non-Gaussian noise can cause severe error propagation in DF approach. In the simulation for the impulse response of optical fiber channels with two scenarios of dc noise and impulsive noises, the proposed method yielded an MSE enhancement of approximately $10 \mathrm{~dB}$ over the linear method. From the results, we can conclude that the proposed method can be an effective candidate for blind signal processing applications from indoor medical devices in $\mathrm{dc}$ and impulsive noise environments to optical fiber communication systems.

\section{REFERENCES}

[1] D. Arnold, "A general noise model and its effects on evolution strategy performance", IEEE Trans. Evol. Comput., vol. 10, no. 4, pp. 380-391, 2006.

[2] E. Kappenman and S. Luck, "The effects of electrode impedance on data quality and statistical significance in ERP recordings", Psychophysiology, vol. 47, pp. 888-904, 2010.

[3] R. Ozawa and K. Iketani, "Electronic endoscope system for reducing random noise of a video signal", U.S. Patent 6900829 B1, May 31, 2005.

[4] P. Chandra, "Measurements of radio impulsive noise from various sources in an indoor environment at $900 \mathrm{MHz}$ and $1800 \mathrm{MHz}$ ", The 13th IEEE International Symposium on Personal, Indoor and Mobile Radio Communications, vol. 2, pp. 639-643, 2002.

[5] K. Blackard, T. Rappaport, and C. Bostian, "Measurements and models of radio frequency impulsive noise for indoor wireless communications", IEEE J. Sel. Areas Commun., vol. 11, pp. 991-1001, 1993. 
[6] S. Miaou, S. Jeng, S. Tsung, C. Hsiao, and T. Lin, "Transmitting capsule endoscope images with wireless LAN and smart antenna systems", Biomed. Eng.-Appl. Basis Commun., vol. 18, issue 5, pp. 246-254, 2006.

[7] T. Hasan-Al-Mahmud, M. Rahman, and S. Debnath, "Performance analysis of best suited adaptive equalization algorithm for optical communication", Journal of Telecommunications, vol. 1, no. 2, pp. 35-41, 2010.

[8] J. Fickers, A. Ghazisaeidi, M. Salsi, G. Charlet, P. Emplit, and F. Horlin, "Decision-feedback equalization of bandwidth constrained N-WDM coherent optical communication sytems", J. Lightwave Technol., vol. 31, pp. 1529-1537, 2013.

[9] L. Garth, "A dynamic convergence analysis of blind equalization algorithms", IEEE Trans. Commun., vol. 49, pp. 624-634, 2001.

[10] J. Principe, D. Xu, and J. Fisher, Information theoretic learning in: S. Haykin, Unsupervised adaptive filtering, Wiley, (New York, USA), pp. 265-319, 2000.

[11] I. Santamaria, D. Erdogmus, and J. Principe, "Entropy minimization for supervised digital communications channel equalization", IEEE Trans. Signal Process., vol. 50, pp. 1184-1192, 2002.
[12] K. Jeong, J. Xu, D. Erdogmus, and J. Principe, "A new classifier based on information theoretic learning with unlabeled data", Neural Netw., vol. 18, pp. 719-726, 2005.

[13] I. Santamaria, P. Pokharel, and J. Principe, "Generalized correlation function: definition, properties, and application to blind equalization", IEEE Trans. Signal Process., vol. 54, pp. 2187-2197, 2006.

[14] N. Kim, H. Byun, and J. Lim, "Blind signal processing algorithms under DC biased Gaussian noise", in Proceedings of SPIE 2013 Nano-bio Sensing, Imaging \& Spectroscopy, Jeju, pp. OC3-5-1-OC3-5-2, 2013.

[15] T. Luo, "Digital equalization of fiber-optic transmission system impairments", M.S. thesis, McMaster University, Canada, 2011.

[16] S. Haykin, Adaptive Filter Theory, Prentice Hall, Upper Saddle River, 4th ed. 2001.

[17] E. Parzen, "On the estimation of a probability density function and the mode", Ann. Math. Stat. vol. 33, p. 1065, 1962.

[18] K. Surachet, "Modeling and analysis of the effects of impairments in fiber optic links", M.S. thesis, Virginia polytechnic institute and state University, United States, 1999.

[19] J. Proakis, Digital Communications, McGraw-Hill, $2^{\text {nd }}$ ed, 1989. 